\title{
Zum Tod von Prof. Dr. Dr. h. c. mult. Heinrich Zollinger Alt-Rektor der ETH Zürich
}

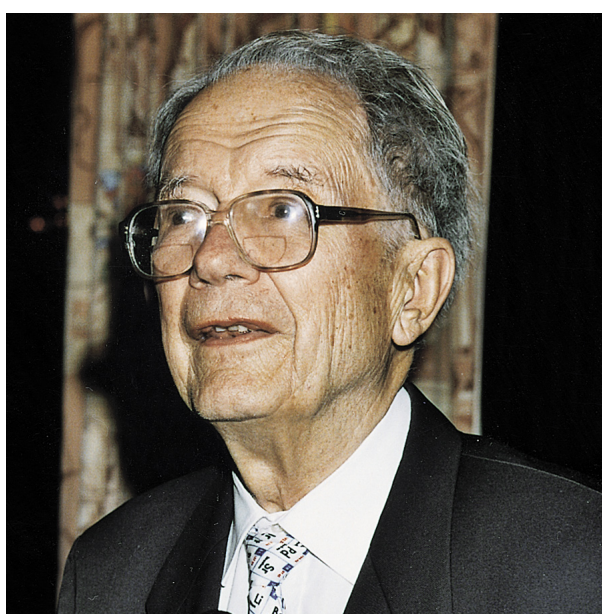

Am 10. Juli 2005 verstarb Heinrich Zollinger in seinem 86. Lebensjahr. Damit verliert die Wissenschaftsgemeinde eine national und international angesehene und verdienstvolle Persönlichkeit.

Nachdem der Verstorbene die Volksschulen in Aarau und Zürich besuchte und das Maturitätszeugnis an der Kantonsschule von Zürich erwarb, studierte er in den Jahren 1939-1943 an der ETH Zürich Chemie. Dort promovierte er 1944 bei Prof. H.E. Fierz-David. Die anschliessende 15-jährige (1945-1960) Tätigkeit in der Farbstoffabteilung der CIBA AG in Basel machte ihn mit der chemischen Industrieforschung wohlvertraut. Während eines Studienaufenthaltes (1951/1952) am Massachusetts Institute of Technology (MIT) in Cambridge (USA) bereitete er sich auf eine neue verantwortungsvolle Aufgabe vor, nämlich auf die Ausbildung des wissenschaftlichen Nachwuchses. Diese Aufgabe bildete von nun an einen der Schwerpunkte seines weiteren beruflichen Wirkens. Seit 1952 lehrte er als Privatdozent an der Universität Basel, bis er 1960 als Assistenzprofessor für organisch-chemische Technologie an die ETH Zürich berufen und 1962 zum ausserordentlichen Professor für Farbstoff- und Textilchemie befördert wurde. Mit grossem Engagement hat sich der Verstorbene dafür eingesetzt, dass das Gebiet der Farbstoff- und Textilchemie an der ETH nicht nur eine stoffliche Behandlung erfährt, sondern dass es den Studierenden von den Grundlagen der anorganischen, der organischen und der physikalischen Chemie her erschlossen wird. Der Erfolg dieser modern konzipierten Lehrtätigkeit manifestiert sich heute in der Tatsache, dass eine bemerkenswert grosse Zahl seiner ehemaligen Studenten, Doktoranden und wissenschaftlichen Mitarbeiter leitende Stellungen in der Industrie innehat oder an namhaften Universitäten lehrt und forscht. Wir alle, die ihn kannten, wissen, mit welch persönlichem Einsatz Prof. Zollinger immer wieder dafür eintrat, dass die Studenten über eine solide und zukunftsgerichtete Ausbildung auf ihr kommendes Berufsleben optimal vorbereitet werden.

Dass dem Verstorbenen aber nicht nur die Ausbildung der Chemiestudenten, sondern auch diejenige aller ETH-Studenten sehr am Herzen lag, zeigte seine aktive Mitwirkung in zahlreichen ETH-internen Kommissionen für Studienfragen (1966-1972). Als es dann im Jahre 1973 darum ging, einen neuen Rektor für die ETH Zürich zu bestellen, war es für viele von uns klar, dass Prof. Zollinger für dieses verantwortungsvolle Amt die notwendige Sachkenntnis mitbrachte. Mit der ehrenvollen Wahl zum Rektor wurde ihm dann für zwei Amtsperioden (1973-1977) eine grosse und nicht immer leichte Aufgabe übertragen, galt es doch auch manche unpopuläre Entscheidung zu treffen. Trotz der grossen Arbeitsbelastung gelang es ihm in dieser Zeit seine Forschungsarbeiten doch noch weiterzuführen.

Die wissenschaftliche Laufbahn des Verstorbenen begann mit seiner Dissertation über Vinylchlorid und dessen Polymerisationsprodukte. Die darauffolgende Industrietätigkeit weckte dann sein Interesse an den Farbstoffen, insbesondere an den Azofarbstoffen. Seine grundlegenden Arbeiten über die Kinetik und den Mechanismus der Azokupplung bildeten die Basis für sein Buch über die „Chemie der Azofarbstoffe“, welches in mehreren überarbeiteten Auflagen erschienen ist und welches heute noch als „Azo-Bibel“ in keinem Farbstofflabor fehlen darf. Prof. Zollinger hat auch in anderen Gebieten der Chemie wertvolle und grundlegende Forschungsarbeiten geleistet. Besonders hervorzuheben sind seine Pionierarbeiten auf dem Gebiet der Reaktivfarbstoffe sowie seine Beiträge über den Reaktionsmechanis- mus elektrophiler und nukleophiler aromatischer Substitutionen. Seine Studien über den Chemismus der Reaktionen von Baumwollfasern mit geeigneten chemischen Verbindungen, wie z.B. mit Reaktivfarbstoffen oder mit Formaldehyd, haben nicht nur wertvolle neue Erkenntnisse auf den Gebieten der Färbemechanismen und der Textilhochveredlung gebracht, sondern auch neue Massstäbe für die wissenschaftliche Erforschung textilchemischer Vorgänge gesetzt. Welches Gewicht der Verstorbene der interdisziplinären Horizonterweiterung beigemessen hat, zeigt auch eine seiner breitangelegten Studien, in welcher er versuchte zu erforschen, ob zwischen der technischen Farbmetrik, der Photochemie und der Neurophysiologie des Farbsehens des Menschen einerseits und der Anthropologie und der Linguistik der Farbempfindung andererseits Zusammenhänge erkennbar sind. Die Erkenntnisse sind in seinem letzten Buch „Color - A Multidisciplinary Approach“ (deutsche Version: „Farbe“, im Druck) zusammengefasst und besprochen. Vielen seiner ehemaligen Doktoranden und Mitarbeitern bleiben aber auch die legendären Gebirgsseminare unvergesslich, in denen nicht nur über laufende Forschungsprojekte in entspannter Atmosphäre nachgedacht, sondern auch über ausserdisziplinäre Fachgebiete referiert und diskutiert wurde.

Es liegt auf der Hand, dass ein so weit gefächertes intensives Schaffen, welches in über 470 Publikationen und zahlreichen Büchern dokumentiert ist, dem Verstorbenen viele nationale und internationale Preise sowie Ehrendoktorwürden namhafter ausländischer Universitäten einbrachte. Ein weiterer Ausdruck nationaler und internationaler Anerkennung für seine Tätigkeit als Forscher, Lehrer und Organisator war seine Wahl zum Präsidenten des Schweizerischen Nationalfonds zur Förderung der wissenschaftlichen Forschung (1979-1982) und zum Präsidenten der Internationalen Union für Reine und Angewandte Chemie IUPAC (1979-1981).

Wir alle, die den Verstorbenen kannten, werden ihm ein ehrendes Andenken bewahren.

Paul Rys 